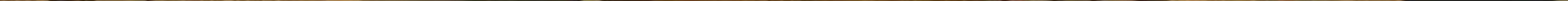




\section{LA RELACIÓN ENTRE LA PRUEBA CEPA Y EL APRENDIZAJE SIGNIFICATIVO}

\section{The relationship between the CEPA test and meaningful learning}

\section{FRANCISCO JAVIER MONTANTES VILLARREAL}

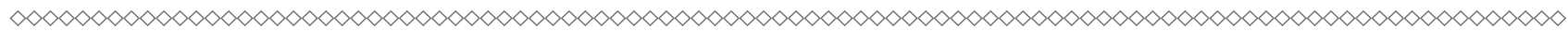

\section{RESUMEN}

El propósito principal de esta investigación es determinar la relación que hay entre los resultados de la prueba CEPA y el aprendizaje significativo en alumnos de cuarto semestre en la modalidad a distancia próximos a graduarse. El método es un estudio tipo no experimental, con un diseño de investigación transeccional. La población estuvo constituida por los 30 estudiantes de cuarto semestre de la modalidad a distancia. Se aplicó el Inventario CEPA para conocer sus intereses, percepciones de motivación, planes; así como su impacto en el aprendizaje significativo manifestado en la acreditación de materia de Información Profesional y Laboral por consiguiente el graduarse de bachillerato. Los resultados se dividieron en los dos grupos de los alumnos que se graduaron y los que no se graduaron, para hacer distinción sobre los que obtuvieron la aprobación de la preparatoria. Se encontró que los graduados prefieren las materias de arte, les desagrada la historia, perciben difícil el español, consideran que tienen buenas calificaciones y sus planes inmediatos son graduarse y en un futuro quieren estudiar y trabajar. A estos datos, se convierte en un perfil de características personales del alumno a distancia graduado, logrando el aprendizaje significativo.

PALABRAS CLAVE: motivación, distancia, graduados, aprendizaje, profesional, prueba.

\section{ABSTRACT}

The main purpose of this research is to determine the relationship between the results of the CEPA test and significant learning in fourth-semester students in the distance mode next to a graduation. The method is a non-experimental study, with a transectional research design. The population was constituted by the 30 students of the fourth semester of the distance modality. The CEPA Inventory was applied to know their interests, perceptions of motivation, plans, etc., as well as their impact on the significant learning manifested in the accreditation of the subject of Professional and Labor Information to obtain the baccalaureate degree. The results were divided into the two groups of students who graduated and those who did not graduate, to make a distinction on those who obtained approval of the preparation. They found that graduates preferred art subjects, they disliked history, they perceived Spanish with difficulty, they had to have good grades and their immediate plans are graduated, in their future they want to study and work. To these data, achieving meaningful learning.

KEYWORDS: motivation, distance, graduates, learning, professional, test. 
E n la Universidad Autónoma de Nuevo León a través del Programa de Educación Media Superior en el año 2003, se realizó la primera aplicación del Inventario CEPA $^{1}$ (Conoce, Explora, Planifica y Actúa) como un recurso de diagnóstico para alumnos de la Generación 2000-2002 de la Preparatoria 8, como plan piloto. Los resultados de la prueba se publicaron en el 2004, en la Revista Presencia Universitaria de la Preparatoria 8, en el Artículo Empleo del Inventario CEPA, donde tiene como eje fundamental "abordar dos perspectivas, una enfocada sobre el interés del alumno por concluir sus estudios y la otra sobre el papel de la Preparatoria en interacción con él". En el mismo se presentan resultados de forma descriptiva del Inventario (CEPA) aplicando al primer y tercer semestre, dentro de la información que destaca "la motivación del alumno es baja y sus habilidades musicales son altas como característica de aquellos alumnos que ya no continuaron sus estudios" (Garza, 2004, pp. 11-12).

En otro artículo plasma la confiabilidad de un cuestionario para rastreo de Trastorno de Aprendizaje (CEPA) en niños en edad escolar, así determinando si puede evaluar dificultades del aprendizaje que facilite la detección de niños que tengan mayor probabilidad de tener trastornos del aprendizaje o como lo llaman también Rendimiento Escolar, fue en una muestra de 681 niños, de escolaridad básica, se tomaron los criterios de inclusión para la prueba piloto, encontrando que "el CEPA es un instrumento adecuado para la evaluación

${ }^{1}$ El Inventario CEPA (CEPA) es un instrumento de orientación que ayuda a los estudiantes de seis a doce años de edad. realizada por el profesor en el aula de clase y de gran utilidad en la investigación" (Gómez, 2010).

En la Preparatoria 2 de la UANL, se desarrolló otra investigación llamada El Uso de Materiales de Multimedia en los Estudiantes, donde su objetivo era determinar la influencia de los materiales de multimedia en el aprendizaje, con un diseño de investigación cuasi experimental con pre prueba, pos prueba y grupos intactos, la muestra fue de tercer semestre en la materia de Ciencias Sociales, se basó éste en los datos de entrada que fueron los registros de los resultados del Instrumento CEPA (2005), encontrando "falta de motivación de los estudiantes para estudiar la materia de Ciencias Sociales" (González, 2010).

En la prueba se contempla la motivación como parte de la medición del estudiante, en este caso, su propia percepción. Es por eso que los procesos motivacionales se relacionan e influyen en la forma de pensar del alumno, las metas que establece, el esfuerzo y persistencia que manifiesta. Por ello que Alonso Tapia (1991) afirma que querer es aprender y saber pensar son las condiciones personales básicas que permiten la adquisición de nuevos conocimientos y la aplicación de lo aprendido de forma efectiva cuando se necesita. Es cierto que existe el plano cognitivo y el proceso de aprendizaje en el plano intelectual, pero aun así existen más procesos motivacionales en los estudiantes.

Así pues, el inventario CEPA es considerado como un instrumento para promover la toma de decisiones y la planificación educativa, ofrece activamente la participación en el proceso de aplicación, recopilación de datos, análisis y uso de la información del mismo, presentando los principales beneficios de la aplicación de este instrumento.

Díaz Barriga (2005) comenta que se entiende que el "logro del aprendizaje significativo está condicionado no sólo por los factores de orden intelectual, sino que requiere como condición básica y necesaria una disposición, deseo o voluntad por aprender", sin la cual todo tipo de ayuda pedagógica estará condenada al fracaso. Hay que reconocer que la motivación actúa como un determinante de la acción, por lo que es importante fomentar y promover la autonomía, el manejo de las emociones, la toma de decisiones y por ende la responsabilidad ante los aprendizajes.

Por otro lado Bañuelos (1993) realiza un estudio para identificar las autopercepciones de habilidades y esfuerzos académicos en una mues- 
tra de estudiantes de nivel superior, en situaciones hipotéticas de éxito y fracaso escolar, encontrando resultados que "muestran diferencias significativas de las auto percepciones de habilidad/esfuerzo de acuerdo con la situación de éxito o fracaso". Indican que un fracaso académico es atribuido a falta de esfuerzo, mientras que el éxito se confiere igual peso a la habilidad y al esfuerzo.

Trataba de conocer ¿Cuál es el efecto de una situación de éxito y fracaso escolar en la autopercepción de habilidad/esfuerzo académico en estudiantes de nivel superior? El instrumento que utilizo fue un Cuestionario de Percepción de Habilidad y Esfuerzo (ante la descripción de una situación hipotética de éxito

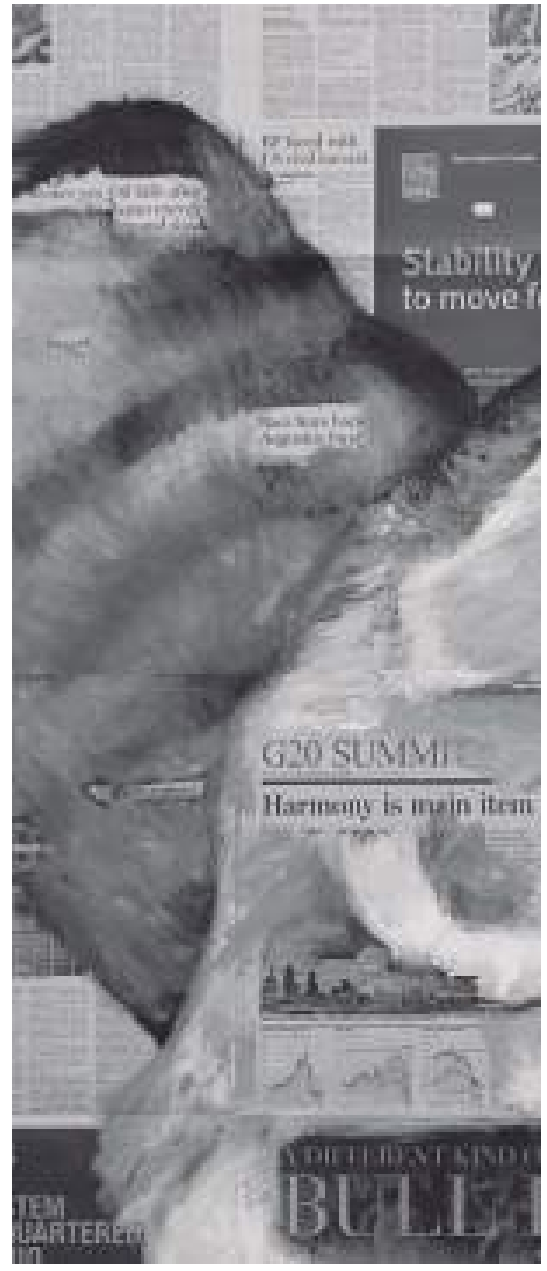

\section{El educado suele distraerse con facilidad, poniéndole menos importancia a lo esencial lo cual es aprender (Alva, 2010).}

o fracaso), aquí el alumno estimó tanto el nivel de habilidad como de esfuerzo académico invertido, eligiendo de cinco opciones, de Mayor a Menor esfuerzo.

En la actualidad existen diferentes distractores en el ambiente de estudio para los adolescentes, como lo es el alcoholismo, las drogas, el internet y las relaciones afectivas y el sexo, según estudios realizados en Perú, país que se encuentra en el

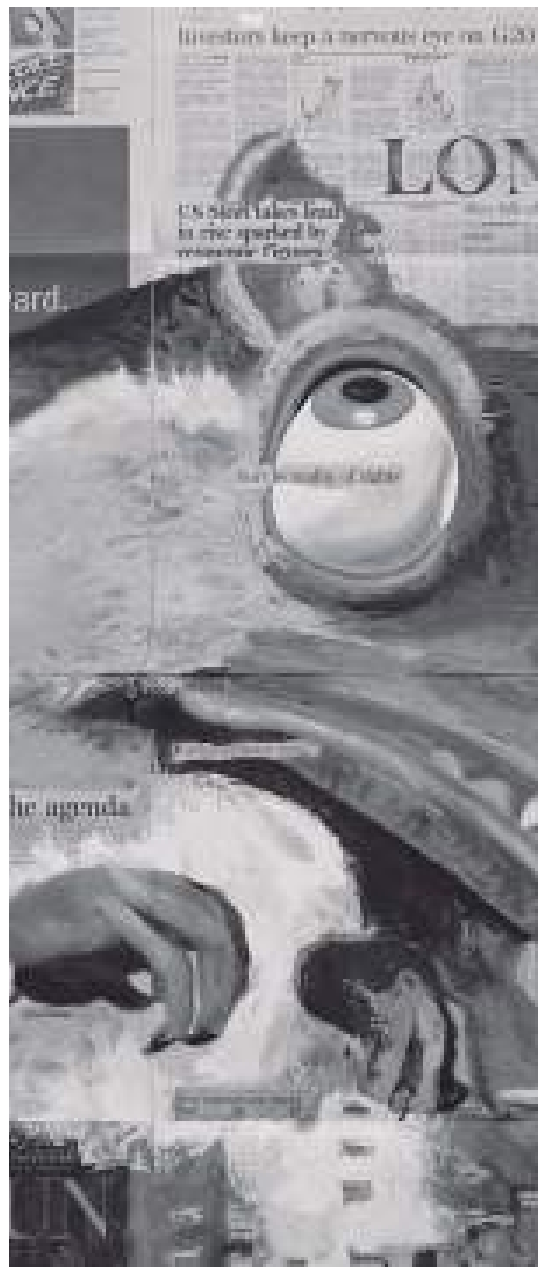

puesto 71 de calidad de educación a nivel mundial, y en el número 7 en Latinoamérica. El educado suele distraerse con facilidad, poniéndole menos importancia a lo esencial lo cual es aprender (Alva, 2010).

Es por eso que en la Educación Media Superior ha enfatizado en un rendimiento académico de alumnos próximo a graduarse en la modalidad de educación a distancia. En el Municipio de Guadalupe la INEGI muestra

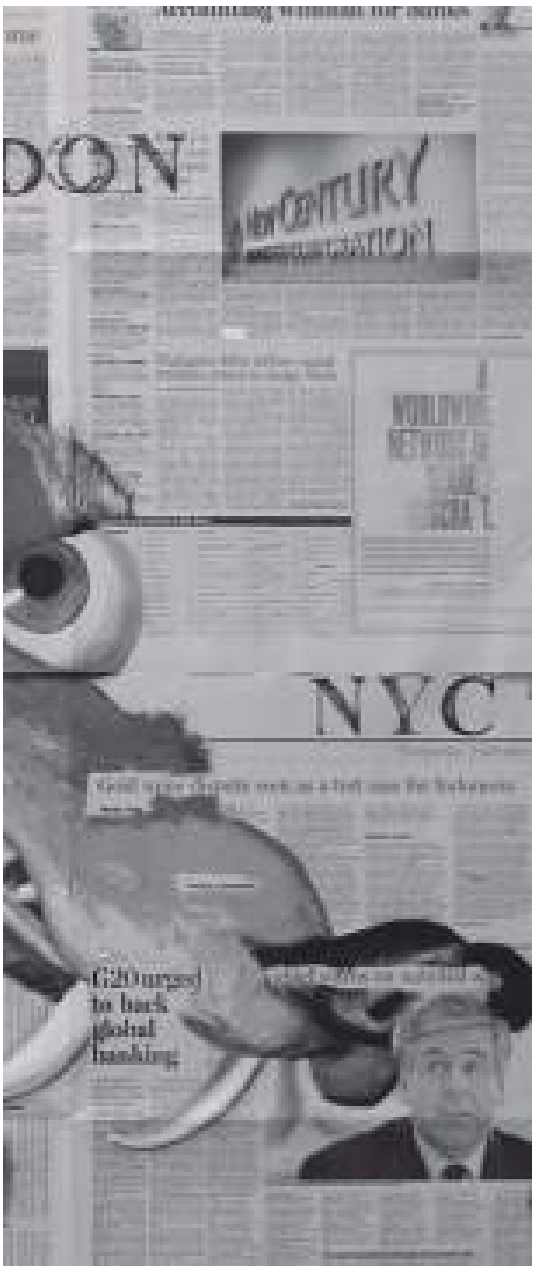


que en el 2006 se presentaron los aspectos geográficos, estado y movimiento de la población e infraestructura, educación y cultura, misma que en su apartado informa sobre los alumnos inscritos, existencia, aprobados y egresados, el índice de retención y aprovechamiento en educación media superior, que conduce a definir en su nivel educativo de bachillerato, "total de alumnos inscritos a la fecha en su publicación de 9,217 de los cuales 4,224 son aprobados y tienen un índice de retención de 95.5\%, su índice de aprovechamiento es de 48.5\%" (CEM, 2007).

Hace aproximadamente seis años inició la modalidad de Educación a Distancia en la Preparatoria No. 8 de la UANL, la cual consiste en cursar el bachillerato de manera semipresencial. La presente investigación pretende realizar un análisis sobre la relación entre la prueba CEPA y el aprendizaje significativo en los alumnos de cuarto semestre con relación al ámbito de reprobación (kárdex) y eficiencia terminal de la institución.

Se realizaron dos investigaciones exploratorias sobre los resultados obtenidos en la aplicación de la prueba del CEPA, llevadas a cabo dentro de los cursos propedéuticos en los periodos escolares 2002-2004 y 2003-2005.

En la primera investigación se aplicó El empleo del inventario CEPA (Conoce, Explora, Planifica y Actúa) avalada por The Collage Board en la creación de programas efectivos en orientación vocacional y tutorías y se encontró que el perfil de ingreso de los alumnos de la preparatoria tienen aspectos como: Intereses ocu-

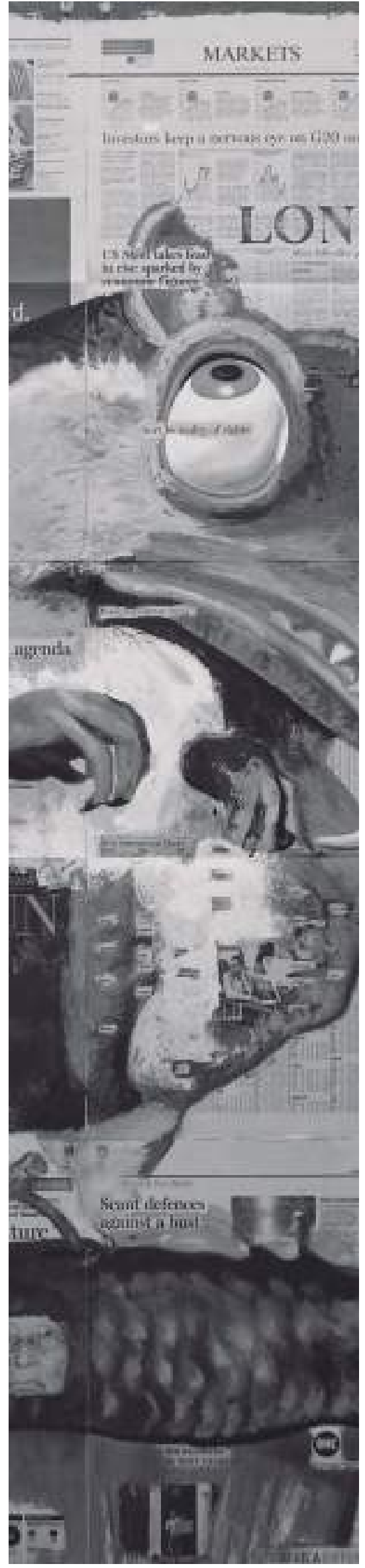

pacionales en el área social, Ambiente de trabajo, gustan de ser creativos y empresariales; Valor Ocupacional en donde su interés más importante es el salario y encontramos que su principal habilidad es el de ayudar y trabajar en equipo (Garza, 2003).

En la segunda investigación Diferencias entre el perfil del desertor y el vigente, detectadas por el CEPA se encontró que las diferencias son en las siguientes áreas: información académica, los planes a futuro, la necesidad de ayuda, sus habilidades, sus valores ocupacionales, los intereses, la percepción académica y su motivación escolar; se concluyó que los alumnos desertores pertenecían al turno nocturno (turno III), quienes tenía una edad por encima de lo normal (16 años), de sexo masculino y no tenían planes a futuro; su principal necesidad de ayuda era en los hábitos de estudio, consideraban que sus habilidades consistía en practicar algún deporte, interés matemático, percepción académica baja y estaban parcialmente motivados (Garza, 2003).

Con la creación del Departamento de Orientación Psicopedagógica de la Preparatoria y su acreditación al Nivel superior ante el Sistema Nacional de Bachillerato, estipulando que para propiciar un servicio integral es necesario que el plantel se acompañe del programa de orientación en los planes de estudio, en este caso de modalidad a distancia, contribuyendo al apoyo individualizado a problemas de aprendizaje y de orientación vocacional, en este caso de Información Profesional y Laboral, con base en los criterios que se establecen de pertinencia, idoneidad y suficiencia. 
La institución realizó un análisis al Departamento de Tutorías en el cual se sistematizó la utilización del CEPA como un instrumento de orientación con el propósito de ayudar a los estudiantes a obtener información relacionada con la toma de decisiones sobre sus estudios y la selección de una ocupación. Éste facilita al estudiante conocer, explorar y actuar con relación a: Intereses Ocupacionales, Planes de Estudio, Preferencias, Habilidades y Tipos de Personalidad.

Un distractor de este grupo de alumnos en su peculiaridad ha sido la propia percepción que tienen de ellos mismo como parte del grupo de educación a distancia, considerando que no forman en su totalidad a la comunidad estudiantil de la misma preparatoria, esta investigación intenta exponer cómo se percibe el alumno de esta modalidad de acuerdo a la prueba CEPA y sus resultados a nivel de aprendizaje significativo (kárdex), manifestado con la aprobación o reprobación de las materias que cursan en el último semestre. Con los resultados del mismo se pretendería tener como parte de su expediente académico y a la vez permita dar un seguimiento de orientación o tutorías para evitar las posibles consecuencias.

\section{OBJETIVO}

Valorar la relación de los resultados del Inventario CEPA en el alumno de bachillerato de la modalidad de educación a distancia con respecto a su aprendizaje significativo mediante el analisis del impacto que tiene la aplicación de estrategias preventivas, formativas y de intervención en la materia de información profesional y laboral.

\section{El Departamento de Tutorías facilita al estudiante conocer, explorar y actuar con relación a: Intereses ocupacionales, planes de estudio, preferencias, habilidades y tipos de personalidad.}

\section{METODOLOGÍA}

Esta investigación de tipo no experimental, se realiza sin manipular deliberadamente las variables, no se hacen variar intencionalmente, aquí se trata de observar fenómenos tal y como se dan en su contexto natural, para después analizarlos, es transeccional ya que se recolectan los datos en un solo momento, descriptivo pues su objetivo es indagar la incidencia y los valores en que se manifiesta una o más variables (Sampieri, 1997).

Estas variables investigadas en una población total, en este caso los alumnos de cuarto semestre de la modalidad a distancia, integrado por 30 alumnos clasificándolo en esta modalidad, siendo de una población total de 1600 alumnos de cuarto semestre en el periodo Enero-Junio del 2015.

Siendo los criterios de inclusión todos los estudiantes que actualmente están cursando el cuarto semestre de bachillerato en modalidad a distancia en la Preparatoria 8 de la UANL, a los cuales se aplicó el Inventario del CEPA, excluyendo aquellos que no recibieron la prueba.

La población en esta modalidad es de 30 alumnos, de los cuales $60 \%$ son hombres (18 de 30$)$ y $40 \%$ son mujeres (12 de 30).

\section{INSTRUMENTO DE INVESTIGACIÓN}

Se utiliza los resultados del Inventario del CEPA, con la finalidad de conocer y explorar a los alumnos de la modalidad a distancia y su aprendizaje significativo, manifestado en el número de materias acreditas del último semestre.

El CEPA (Conoce, Explora, Planifica y Actúa) es un instrumento de orientación - no es un examen- desarrollado en el 2000, dirigido a alumnos de bachillerato, con el propósito de ofrecer información a los alumnos sus padres y las escuelas para facilitar la toma de decisiones y la planificación educativa y ocupacional, se fundamenta las bases teóricas del desarrollo ocupacional.

El consentimiento informado para obtener los datos de la aplicación del instrumento fue otorgado por las autoridades escolares a través del Departamento de Orientación y Tutorías de la Preparatoria 8 UANL.

\section{PROCEDIMIENTO}

La elaboración de esta investigación se pudo llevar a cabo a través del siguiente procedimiento de recolección de datos: 
TABLA 1. Porcentaje de Alumnos que se Graduaron.

\section{ALUMNOS QUE SE GRADUARON \\ FRECUENCIA \\ PORCENTANE}

Si

No

Total
21

9

30
$70 \%$

$30 \%$

$100 \%$
1. Obtener el listado completo de los alumnos inscritos en el tercer semestre del periodo Agosto -Diciembre de 2014, en la modalidad a distancia.

2. Capacitar a los Profesores que pertenecen a la Academia de Orientación para la aplicación del CEPA en la materia de Información Profesional y Laboral.

3. Aplicar el inventario del CEPA a los alumnos inscritos en la semana que se programe por la Dirección de Preparatoria, de acuerdo al listado de alumnos de educación a distancia.

4. Enviar a Centro de Evaluación de la UANL las hojas de respuesta correspondiente al periodo de Agosto-Diciembre del 2014.

5. Solicitar los resultados de la aplicación de la prueba a Centro de Evaluación a través del oficio correspondiente.

6. Recepción de resultados del CEPA en el cuarto semestre, periodo Enero-Junio del 2015.

7. Clasificación de los instrumentos para su entrega, correspondiente a las listas de asistencia a los grupos, obteniendo a los alumnos que no continuaron y los que si continuaron, con apoyo del Departamento de Orientación de la Preparatoria.

8. Recolección y clasificación de la prueba a través del Excel, con los datos del alumno e incluyendo en el mismo el check list.

9. Realizar la captura de la información generada a través de la aplicación del Inventario del CEPA.

10. Obtener la estadística en el sistema SSPS.

\section{RESULTADOS}

Al terminar de capturar los datos en la base en el sistema SPSS', se procedió a la verificación del total de los instrumentos aplicados, ya que no hubo ninguno de estos sin contestar para considerarlo nulo, por lo que la muestra final fue completa. Cabe recordar que los datos generales y escolares fueron recopilados en el Departamento de Orientación, mientras que los datos referentes a los aspectos de graduados y no graduados fueron a través de Departamento de Servicios Escolares.

En esta parte se presentan los resultados obtenidos de cada una de las 12 respuestas del CEPA, de acuerdo al análisis elaborado, esto permitirá responder algunos objetivos de la investigación realizada.

El primer análisis realizado fue de tipo descriptivo con la finalidad de averiguar la ocurrencia en sus características como alumno de la modalidad a distancia que se graduaron y no se graduaron. En esta parte se presentan los resultados obtenidos de cada una de las respuestas del CEPA, de acuerdo al análisis elaborado, esto permitirá responder algunas cuestiones de la investigación realizada.

Los resultados para el primer grupo de variables - las relacionadas con las características personales de los estudiantes- basándose en la percepción que tiene el alumno con respecto a las materias, los planes, intereses, valores ocupacionales, ambientes de trabajo y su propia motivación. También la acreditación de la materia de Información Profesional y Laboral con relación de los alumnos que se graduaron y no se graduaron.

\section{CONCLUSIÓN}

La relación entre la prueba CEPA y el aprendizaje significativo en los alumnos de cuarto semestre, reflejado en la graduación de estos en la modalidad a distancia, a los alumnos les gustan la materia de artes, no les agrada la historia, consideran la materia más difícil el español, se perciben como alumnos que obtienen buenas calificaciones, sus planes inmediatos son graduarse de bachillerato, en un futuro quieren estudiar y trabajar y a largo plazo quieren graduarse de licenciatura, su interés

${ }^{2}$ SSPS: es un programa estadístico informático muy usado en las ciencias sociales. 


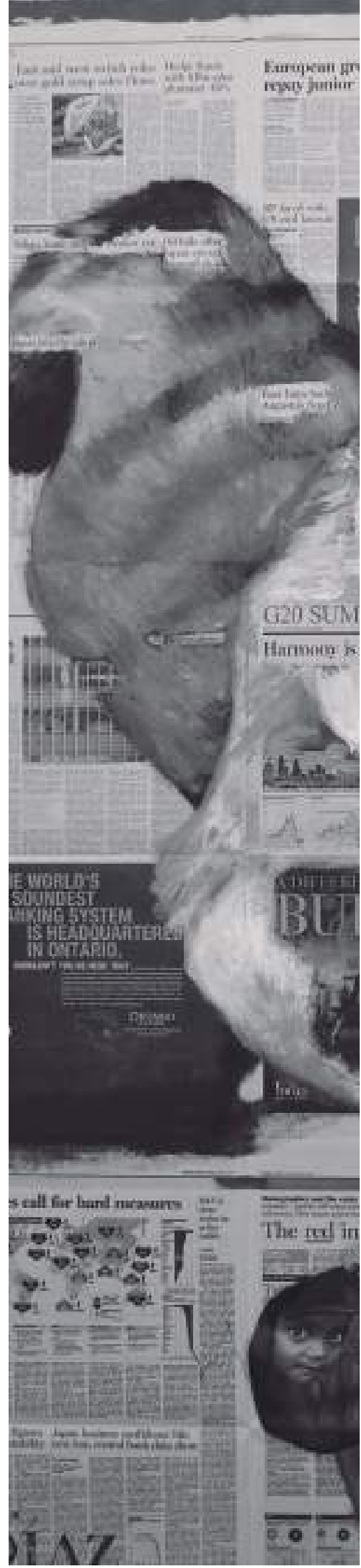

\section{Se obtuvo un perfil de los graduados} en la modalidad a distancia formado por las características generadas por el CEPA. Reflejando su aprendizaje significativo en el logro de la acreditación de las materias de cuarto semestre.

ocupacional más alto es el creativo y social, el más bajo es el de oficina, su valor ocupacional es la actividad física, prefieren el ambiente de trabajo científico y empresarial, se encuentra motivado. Por último los alumnos que se graduaron aprobaron la materia de Información Profesional y Laboral.

Se obtuvo un perfil de los graduados en la modalidad a distancia formado por las características generadas por el CEPA. Reflejando su aprendizaje significativo en el logro de la acreditación de las materias de cuarto semestre.

Dentro de las estrategias preventivas es la utilización de un instrumento para diagnosticar un perfil de alumno graduado, así también el seguimiento de la atención a los alumnos de cuarto semestre a través de las sesiones de la materia de Información Profesional y Laboral.

La contribución del presente estudio es una propuesta de atención personalizada a los estudiantes próximos a graduarse, utilizando los resultados de los instrumentos estandarizados como lo es el CEPA. Además de hacer mejoras continuas a las estrategias de seguimiento realizadas en la ma- teria de Información Profesional y Laboral creando una parte innovadora para próximos alumnos de la modalidad a distancia.

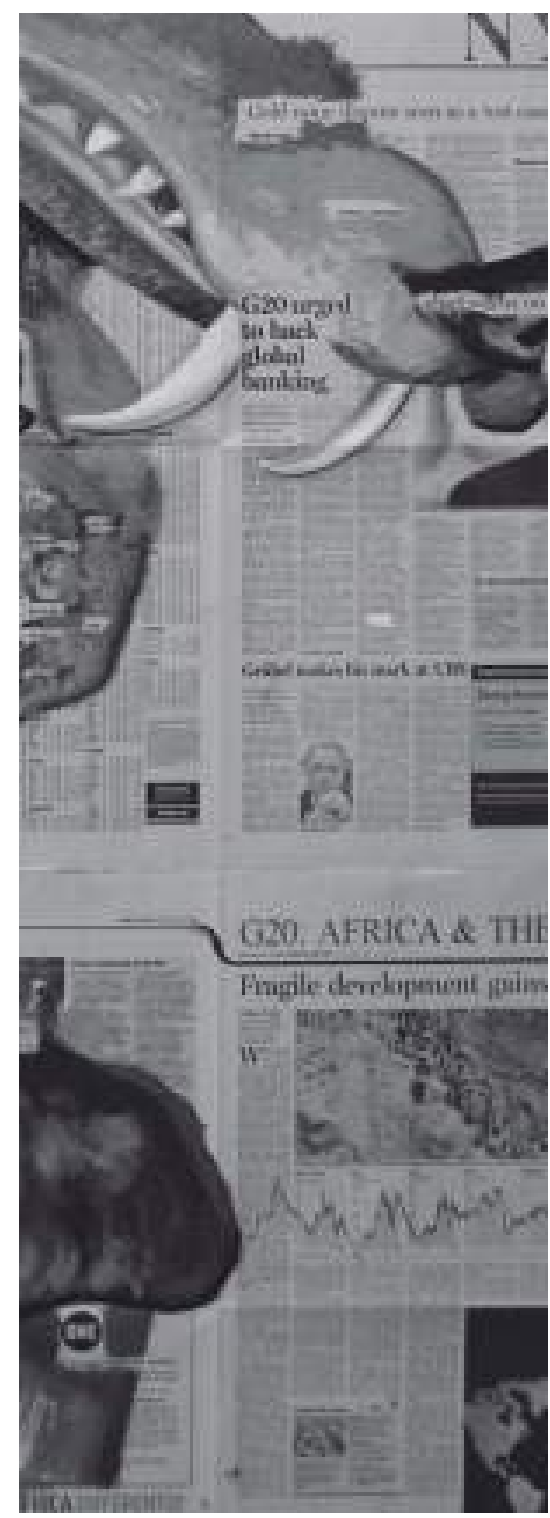




\section{REFERENCIAS}

Alonso, T. Jesús. Motivación para el aprendizaje: la perspectiva de los alumnos. Facultad de Psicología. Universidad Autónoma de Madrid. 209 -238. https://books.google.com.mx/ books?hl=es\&lr=\&id=ilPd3G7gA_cC\&oi=fnd\&pg=PT210\&dq=APRENDIZAJE+SIGNIFICATIVO+Y $+P E R C E P C I O N+D E+L A+M O T I V A C I O N \&$ ots=iCBm4qnWW\&sig=PgYym4bk5Yw_KsLnLWUUhXglP LI\#v=onepage \&q=APRENDIZAJE\%20SIGNIFICATIV0\%20Y\%20PERCEPCION\%20DE\%20LA\%20 MOTIVACION\&f=false Fecha de consulta Abril del 2016.

Alva, H. Ana. (2010) Principales distractores Universitarios. http://distractoresuniversitarios. blogspot.mx/ Fecha de consulta Abril del 2016.

Bañuelos, M. Ana María. Perfiles Educativos. Motivación Escolar: Estudio de variables efectivas. http://www.redalyc.org/html/132/13206011/ Fecha de consulta Enero del 2016.

Comisión Nacional de la Mujer. Septiembre, 2007. El enfoque de género en la producción de las estadísticas educativas de México: una guía para usuarios y una referencia para productores de información.

Díaz Barriga, F. Estrategias Docente para el aprendizaje significativo, Tercera Edición. McGraw Hill. Capítulo 3, 96 -110.

Garza, Elizabeth. (2004). Empleo del Inventario CEPA (Conoce, Explora, Planifica y Actúa). Presencia Universitaria, 26(5), 11-17.

Gómez, B., Romero G., Aguirre D. Confiabilidad de un cuestionario para rastreo de trastorno de aprendizaje (CEPA) en niños en edad escolar. Universidad San Buena Aventura, Volumen 10, No. 1. P-1-16. (2010).. http://www.revistas.usb.edu.co/index.php/Agora/article/view/364/124

González, Laura. El uso de materiales multimedia en los estudiantes de la Preparatoria No. 2 de la Universidad Autónoma de Nuevo León. Revista Investigación e Innovación Educativa en el Estado de Nuevo León. Memorias REDIIEN 08. Enero 2010. Consulta Enero 2016. https://www.researchgate. net/profile/Alma_Gomez_Galindo/publication/273774043_Investigacin_e_Innovacin_Educativa_ en_el_Estado_de_Nuevo_Len._Memorias_REDIIEN_08/Links/550c8c6f0cf2752610964c64. pdf\#page=33

Sampieri, R. Metodología de la investigación.,(1997), 2da Edición, Impresora OFGLOMA, S.A. México D.F 


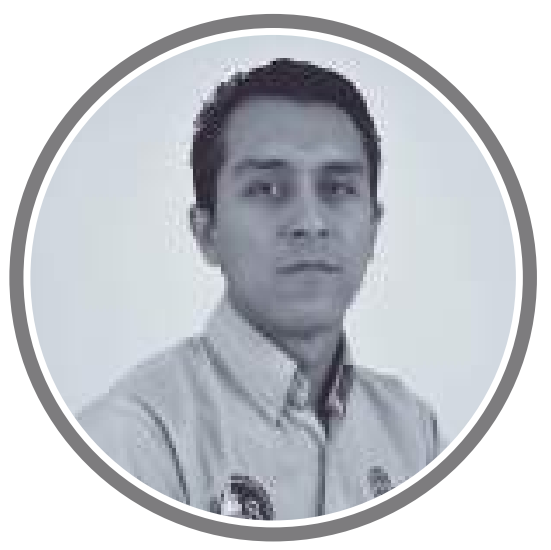

\section{Francisco Javier Montantes Villarreal}

Es egresado de la Facultad de Psicología UANL del área conductual. Posee el grado de Maestro en Docencia con Orientación en Educación Media Superior por la misma facultad con la tesis $L a$ relación entre la prueba cepa y el aprendizaje significativo en los alumnos de cuarto semestre de bachillerato en la modalidad a distancia. Es profesor certificado en competencias docentes del Nivel Medio Superior y con Nivel 5 otorgado por el Sistema de Capacitación y Certificación en el Deporte. Actualmente es Secretario Administrativo en la Preparatoria 8 y Docente del Cuerpo Académico Disciplinar Ciencias Experimentales.

Correo electrónico:

franco_javier_97@hotmail.com

Recibido: 20/11/2018

Aceptado: 20/02/2019 